\title{
Dissecting the Effects of Concentrated Carbohydrate Solutions on Protein Diffusion, Hydration, and Internal Dynamics
}

\author{
Enrico Spiga, ${ }^{\dagger} \S$ Luciano A. Abriata, ${ }^{\dagger}, \S$ Francesco Piazza, ${ }^{\ddagger}$ and Matteo Dal Peraro ${ }^{* \dagger}$ \\ ${ }^{\dagger}$ Laboratory for Biomolecular Modeling, Institute of Bioengineering, School of Life Sciences, École Polytechnique Fédérale de \\ Lausanne (EPFL), and Swiss Institute of Bioinformatics (SIB), 1015 Lausanne, Switzerland \\ ${ }^{\ddagger}$ University of Orléans and Centre de Biophysique Moléculaire (CNRS-UPR 4301), Rue C. Sadron, 45071, Orléans, France
}

Supporting Information

\begin{abstract}
We present herein a thorough description of the effects of high glucose concentrations on the diffusion, hydration and internal dynamics of ubiquitin, as predicted from extensive molecular dynamics simulations on several systems described at fully atomistic level. We observe that the protein acts as a seed that speeds up the natural propensity of glucose to cluster at high concentration; the sugar molecules thus aggregate around the protein trapping it inside a dynamic cage. This process extensively dehydrates the protein surface, restricts the motions of the remaining water molecules, and drags the large-scale, collective motions of protein atoms slowing down the rate of exploration of the conformational space despite only a slight dampening of fast, local dynamics. We discuss how these effects could be relevant to the function of sugars as preservation agents in biological materials, and how crowding by small sticky molecules could modulate proteins across different reaction coordinates inside the cellular cytosol.
\end{abstract}

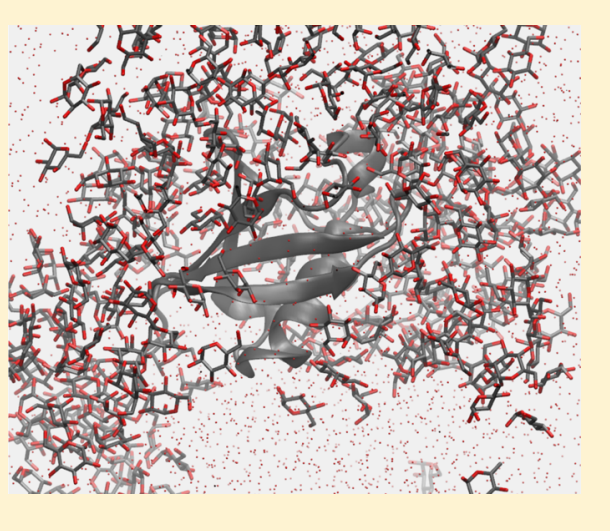

\section{INTRODUCTION}

Sugars play roles as agents for the preservation of biological material in nature and in biotechnological manufacture, ${ }^{1-3}$ most importantly through their capacity to stabilize proteins against cold and hot denaturation, both in solution and in the solid state. ${ }^{4-7}$ Other potential effects of sugars on protein properties have been less explored, but changes in activity, dynamics, and regulation can be expected by analogy to the effects known to be caused by high concentrations of other molecules. Whereas most hydrophilic molecules have the capacity to agglomerate and disrupt water structure, sugars and polyols generally have exceptionally large solubilities, which allows them to strongly dehydrate other molecules and to cluster at very high concentrations forming glassy states. ${ }^{8-11}$ Because of these special properties, questions about the effects of sugars on protein properties are intimately related to those revolving around the effects of viscosity, molecular crowding, encapsulation and even vitrification on proteins, all meeting at the crossroads between chemistry, biology, medicine, and applications in the food and pharmacological industries. Within all these closely related fields, the effects of high concentrations of small hydrophilic molecules on protein stability and translational and rotational diffusion have been explored, but studies of their effects on protein hydration and on internal protein dynamics are scarce. ${ }^{4-7,12-16}$ More specifically to sugars, reports on the structure and dynamics of sugar-only solutions abound, ${ }^{8,17-23}$ but works dealing with proteins in sugar solutions are mostly focused on the stability of the proteins and do not pay much attention to other effects related to hydration, diffusion, and internal mobility. ${ }^{4-7}$ We recently reported in a preliminary work that concentrated glucose solutions can perturb protein dynamics by restricting exploration of the conformational space, through a mechanism that presumably involves protein-sugar interactions. ${ }^{16}$ However, we could not provide at that time details on why and how the exploration of the conformational space is altered, what the effects on hydration structure and dynamics are, how glucose's propensity to cluster relates to these processes and what the practical outcomes of all these effects could be. In the present work, we attempted to assess these points and to fully dissect the effect of high glucose concentrations on protein properties by describing results from molecular dynamics (MD) simulations in several systems containing one or more protein molecules and a large numbers of glucose molecules that form a crowded, concentrated solution of this small, very hydrophilic molecule.

Within the scope of biological chemistry, glucose falls in the category of small hydrophilic solutes, which make up around $25 \%$ of the $300-400 \mathrm{~g} / \mathrm{L}$ solutes found inside the highly crowded cellular environments. ${ }^{24}$ Thus, beyond the intrinsic relevance of glucose given its abundance in biological systems and its applications in biotechnological manufacture, studies of proteins in concentrated glucose solutions can in principle help to unveil the effects of small hydrophilic crowders inside cells. Such crowded conditions are very important in shaping life at the molecular level, since they force molecules into unspecific

Received: December 27, 2013

Revised: April 23, 2014

Published: April 28, 2014 
interactions that alter diffusion through the cell, the structures and internal dynamics of biomolecules, their activity and interaction capabilities, and so on, ultimately affecting the equilibria and cellular processes in which these macromolecules are involved. ${ }^{12-15,25-44}$ These effects are so relevant that it is being increasingly recognized that they should be, and are in fact being, accounted for in experimental and computational studies dealing with biomolecules. ${ }^{12,29,39,40,45-58}$

Probably the most studied effects of viscosity, molecular crowding, and encapsulation on protein properties are those related to the dampening of translational and rotational diffusion ${ }^{15,30-32}$ and those linked to changes in stability. $^{4-7,28,33,35,36,59-64}$ More recently, experiments and simulations suggested that these conditions can also influence hydration and internal protein dynamics, ${ }^{16,37,40,58,59,65,66}$ which are essential for proteins to function. However, the inherent difficulty in exploring protein dynamics and hydration, especially under crowded, encapsulated, and high-viscosity conditions, has so far hampered detailed studies on these aspects. The few insights available to date arose from studies on ubiquitin, a small protein whose structural dynamics (i.e., conformational fluctuations) are very important for recognition of its different protein targets. Indeed, ubiquitin ensembles refined against NMR data about motions on the nanosecondsmicroseconds time scale show that the protein explores in solution a landscape that encompasses most of the conformations in which it is bound to other proteins. In turn, MD simulations show that this landscape contains three main basins that are crossed multiple times in the long nanoseconds time scale, two of them very close, as seen in our simulations, and three of them with small structural differences that map mainly to three loops (shown in Figure S1 together with some more information).

Ubiquitin dynamics have been vastly studied through experiments and simulations in dilute solution ${ }^{67-74}$ and more recently under crowded-like conditions. First, two related works reported that confinement of ubiquitin inside reverse micelles, which can be related to crowded conditions, slightly reduces its picoseconds-nanoseconds flexibility, although motions in slower time scales were not probed. ${ }^{45,66}$ We then reported that exploration of ubiquitin's conformational space in submicrosecond MD simulations was impaired in systems set up at high glucose concentrations, compared to reference conditions in water. ${ }^{16}$ This interesting observation generated novel hypotheses and raised a number of issues that we address here. Does this glucose-crowded condition affect conformational diffusion through a conserved landscape, or does it also alter the shape of this landscape? How are motions on different time scales differentially affected? How do changes in internal dynamics relate to the better understood changes in translational and rotational diffusion? To what extent is protein hydration affected? Are there any relationships between the observed effects and glucose's clustering and desolvating capacities?

In order to answer these questions, headed towards dissecting the effects of small hydrophilic molecules on protein properties, we have probed the effect of increasing glucose concentrations on unbiased $\mathrm{MD}$ and replica-exchange $\mathrm{MD}$ (REMD) simulations of ubiquitin. We have studied systems set up with one or three copies of the protein in different starting conformational states, in either water or glucose solutions at 108 or $325 \mathrm{~g} / \mathrm{L}$ concentration (Table S1). The choice of glucose for these studies relies on the facts that (i) it is abundant in nature and in man-made products, (ii) it has been much studied to understand its clustering and percolating properties and how it stabilizes proteins, and (iii) it can be accurately modeled in atomistic simulations even reproducing quantitatively the formation of clusters and desolvation potential at high concentrations. $4,7,8,17-19,22,75$ In all our simulations, we used fully atomistic descriptions of all the systems, as required to capture not only mere steric effects but also realistic interactions between the different components.

The simulations on systems containing a single protein molecule and glucose are all $1 \mu \mathrm{s}$ long, allowing us to dissect the effect of the concentrated small molecule on the protein's flexibility in different time scales, its diffusion properties, and perturbations on the structure and mobility of water and glucose molecules around the protein surface. From these analyses we conclude that the protein acts as a seed for clustering of glucose molecules to occur around the protein, as predicted from theory in a previous work, ${ }^{8}$ leading to the formation of a dynamic cage of glucose molecules around the protein and to strong desolvation of its surface. This perturbs translational and rotational diffusion, but of most novelty, we observe that this slowdown is transmitted to the protein interior so that, whereas fast, local dynamics are reduced only slightly under crowded conditions, collective motions are strongly dampened, with a predicted slowdown larger than that observed for translational and rotational diffusion. Finally, 250 ns-long simulations on systems containing three protein molecules allow us to visualize that the cages of glucose molecules around the proteins project into the solution and contact each other. This results in the formation of structures that bridge the glucose cages surrounding different proteins, much like the "noncovalently interacting metabolite structures" proposed by Cossins et al. in their simulation of the bacterial cytosol. $^{29}$ This supports the growing hypothesis of the cytoplasm being a biphasic gel-sol system ${ }^{76}$ with the additional insight that small hydrophilic molecules could provide the driving force for phase separation.

\section{METHODS}

Molecular Dynamics Simulations. Unbiased MD simulations were run with the $\mathrm{NAMD}^{77}$ code using the amber99SB ${ }^{78}$ force field for the protein, TIP3P ${ }^{79}$ for water and Glycam $06^{80}$ parameters for $\alpha$-D-glucose (we have modeled the alpha anomer only, but given the very similar physicochemical properties of the alpha and beta anomers this choice should have minor consequences on the effects that we studied). NPT conditions were set at $1 \mathrm{~atm}$ and $300 \mathrm{~K}$. The structure of ligand-free human ubiquitin in PDB ID $1 \mathrm{D} 3 \mathrm{Z}^{81}$ was minimized and equilibrated in water to produce the conformation that corresponds to state $\mathrm{C}$ in Figure 1A. Starting states $\mathrm{L}$ and $\mathrm{R}$ were extracted from stable sections of the simulation of state $\mathrm{C}$ in water, lasting for more than $10 \mathrm{~ns}$ around the minima in the PCA frame shown in Figure 1A. Simulation boxes were built by combining the corresponding protein structures together with a random distribution of glucose molecules using the $\mathrm{Packmol}^{82}$ program, and then solvated with TIP3P water molecules. Further details about these simulations are given in the Supporting Information (SI) text.

REMD simulations ${ }^{83}$ were run with the AMBER code ${ }^{84}$ using the same structures and parametrizations described above for protein, water, and glucose molecules. The simulation in water was $30 \mathrm{~ns}$ long, and those with glucose were $70 \mathrm{~ns}$ long. 
All of them were run in NVT conditions with a Langevin thermostat as shown to be optimal by Rosta et al. ${ }^{85}$ Further details about these simulations are given in the SI. For each simulation, 48 replicas were computed at temperatures between 290 and $389 \mathrm{~K}$ (all temperatures listed in the SI) and exchanges were attempted every 1 ps as suggested by Sindhikara et al. ${ }^{86}$ The analyzed data corresponds to all sections run at $301.1 \mathrm{~K}$, joined together for each system. We have not used unbiasing techniques to reconstruct the energy landscape, as we only intended to see with these simulations whether the proteins could escape their minima. We provide an assessment of these simulations in the SI (Figure S11 and Table S6) mostly based on analyses described by Sindhikara et al. ${ }^{86}$

Projection and Analysis of the MD Trajectories. The reference frame employed for projecting the simulated trajectories was built through principal components analysis of the covariance matrix of $\mathrm{C} \alpha$ positions for residues $2-70$ as retrieved from 72 high-resolution X-ray structures of human ubiquitin aligned to model 1 of PDB ID 1D3Z. ${ }^{81}$ Projection of the trajectories on the reference frame was also preceded by alignment to this reference structure. Logarithmic probability distributions were built by projecting the trajectories on a reference frame, binning them into a grid and counting the number of frames inside each cell of the grid $\left(N_{i}\right)$ to compute $-k_{\mathrm{B}} T \ln \left(N_{i} / N_{0}\right)$, where $N_{0}$ corresponds to the most populated bin thus setting the offset. The method is basically the same that Lange et al. introduced, ${ }^{72}$ plus the calculation of logarithmic densities that Long et al. ${ }^{90}$ employed to visualize the projected trajectories.

The MD trajectories were analyzed in terms of the rootmean squared fluctuations of $\mathrm{C} \alpha$ atoms (RMSF), order parameters of $\mathrm{N}-\mathrm{H}$ bonds $\left(\mathrm{s}^{2}\right)$, mean square displacements of $\mathrm{C} \alpha$ atoms (MSD), translational diffusion coefficients and rotational relaxation dynamics of the protein, the mechanical description of the conformational basins and the dynamics of water and glucose molecules interacting with the protein surface. The mechanical description of conformational basins was achieved using a formulation developed by Hess, ${ }^{87}$ which characterizes each basin in terms of an harmonic force constant $k$ and an internal friction-like coefficient $\eta$ for each principal component of motion inside the basin. Interactions between water or glucose molecules and the protein were investigated by computing and fitting the survival probabilities of their contacts with protein residues, as reported in previous formulations. ${ }^{88,89}$ Details on how these analyses were performed are given in the SI.

\section{RESULTS}

Ubiquitin dynamics have been vastly studied through experiments and simulations, disclosing the importance of collective motions in producing conformations that resemble those observed in different complexes of the protein bound to its targets. As shown in many works, 0.5 to $1 \mu \mathrm{s}$-long $\mathrm{MD}$ simulations of ubiquitin can reproduce NMR order parameters and residual dipolar couplings very well, indicating good agreement between simulations and experimental observables. ${ }^{16,69,72,90}$ Also, such simulations reproduce local and large-scale conformational fluctuations observed in X-rays structures. The latter are easily visualized by projecting the MD trajectory on a two-dimensional plane built from available $\mathrm{X}$-rays structures as shown in a number of recent works. ${ }^{72,90-92}$ Figure 1A shows such a projection for a 600 ns-long simulation of ubiquitin in water, relative to 72 structures used to build the reference frame and converted into a logarithmic probability distribution as described under methods. Segments of ubiquitin that experience collective conformational variability according to the PCA analysis correspond to residues 6-11, 33-36, and 46-49 as shown in Figure 1B.

The conformational landscape of ubiquitin simulated in water displays two main basins: a smaller one centered at around $[-4.5,0.5] \AA$ in the reference space, and a larger one centered at around $[0,0] \AA$ (Figures $1 \mathrm{~A}$ and $2 \mathrm{~A}$ ). Based on the

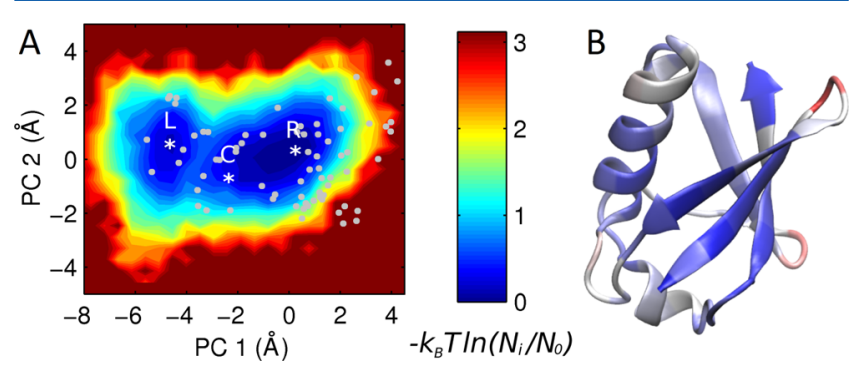

Figure 1. Conformational space and flexibility of ligand-free ubiquitin in water. (A) Conformational landscape (color-coded as a logarithmic probability distribution) explored by ubiquitin during a $600 \mathrm{~ns}$-long simulation in water, as derived from its projection on the 2 first principal components which describe $42.6 \%$ and $12.5 \%$ of the variability observed in X-ray structures (gray points). Asterisks denote starting states L, C, and R for simulations under glucose-crowded conditions. (B) The flexible segments of ubiquitin (increased flexibility from blue to red) as revealed from the analysis of variation in X-ray structures.

shape of this landscape and on our previous results, ${ }^{16}$ we defined three relevant conformations for subsequent simulations: state L (after "left") corresponding to the deepest point of the smallest basin, state C (after "center") corresponding to the structure of ligand-free ubiquitin employed for the simulation in water, and state R (after "right") corresponding to the deepest point of the largest basin. Placing two starting states on the larger basin serves two purposes: first, in some simulation studies this large basin is actually seen as two basins separated by a very small energy barrier (with minima approximately on states $\mathrm{C}$ and $\mathrm{R}$ ) ${ }^{90}$ second, a starting state at $\mathrm{C}$ corresponds to static structures of unbound ubiquitin. In what follows, we compare simulations of ubiquitin dynamics in water, $108 \mathrm{~g} / \mathrm{L}$ glucose and $325 \mathrm{~g} / \mathrm{L}$ glucose, started from structures that correspond to these different initial states.

Effect of Increasing Glucose Concentration on the Dynamics of Ubiquitin. In a free MD simulation of ubiquitin in water, in only $40 \mathrm{~ns}$ the protein has already explored most of the conformational space that it will explore in the rest of the simulation (Figure 2A) and that it is known to explore in microsecond-long simulations. ${ }^{69,72,90}$ Instead, the projections of the simulation in $108 \mathrm{~g} / \mathrm{L}$ glucose (Figure $2 \mathrm{~B}$, center) show that after $500 \mathrm{~ns}$ the protein is stuck in what corresponds roughly to the rightmost basin of the simulation in water. After $1 \mu \mathrm{s}$ of simulation, the protein has sampled also part of the leftmost basin, albeit incompletely. When the glucose concentration is raised to $325 \mathrm{~g} / \mathrm{L}$, the situation is more extreme, with only one basin visited that corresponds to the left area of the rightmost basin observed in water (Figure 2C, center). These observations predict that crowding slows down conformational sampling. However, we cannot say from these simulations whether the crowded conditions are wiping out the 

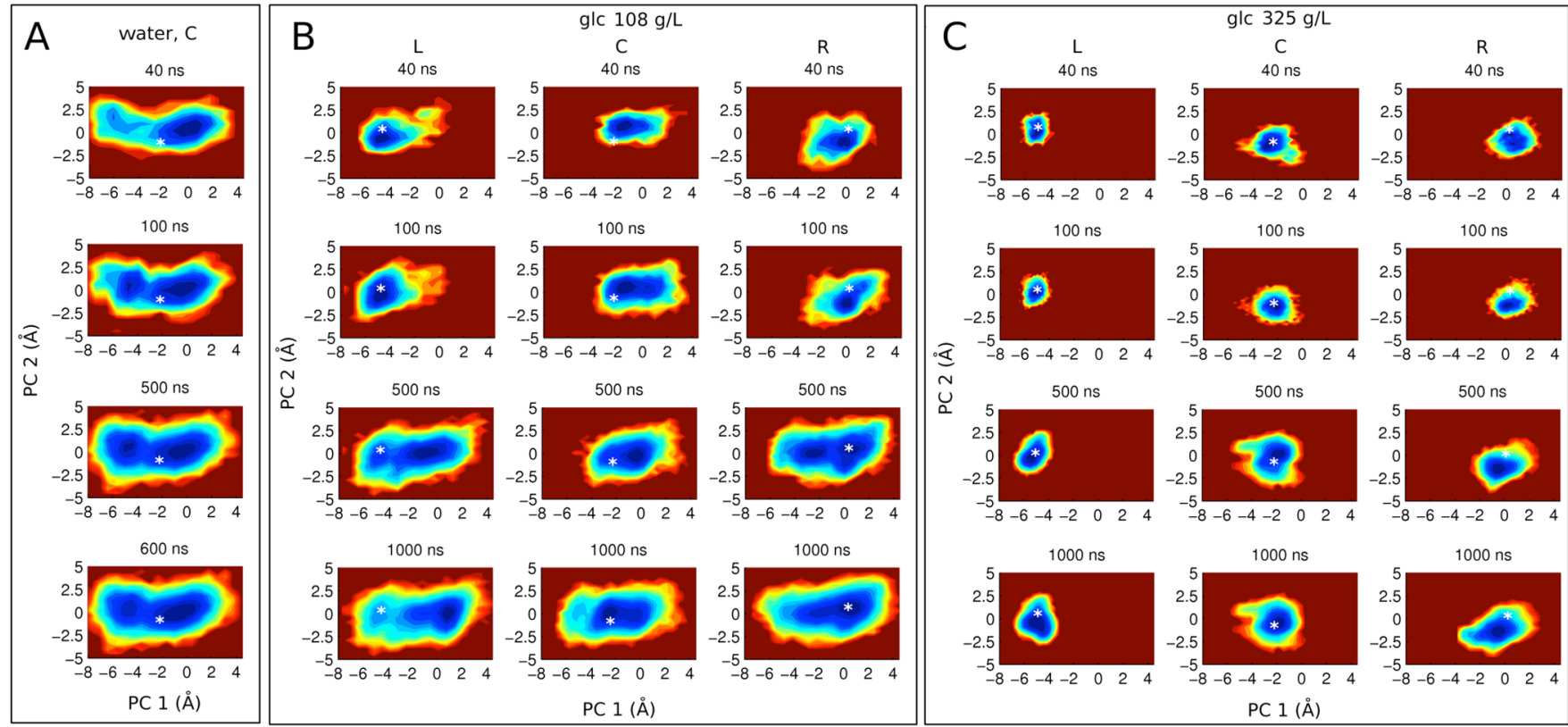

$100 \mathrm{~ns}$
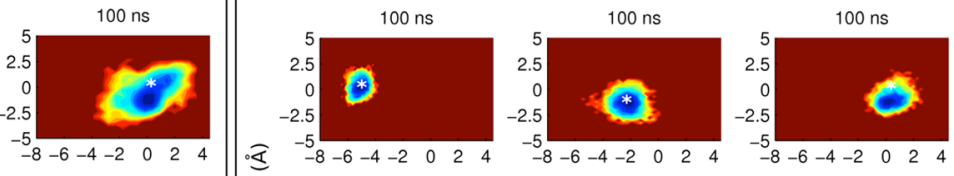

$500 \mathrm{~ns}$
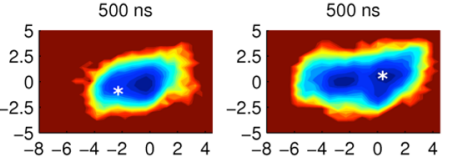

$\stackrel{\circ}{\sim}$

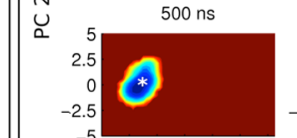

$\begin{array}{rr}-8-6-4-2 & 0 \\ 5 & 500 \mathrm{~ns}\end{array}$
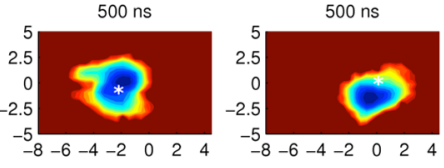

$1000 \mathrm{~ns}$

$1000 \mathrm{~ns}$

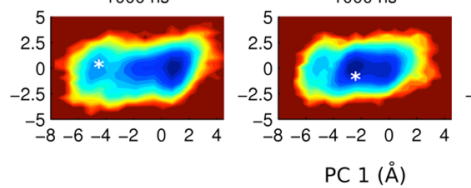

$1000 \mathrm{~ns}$
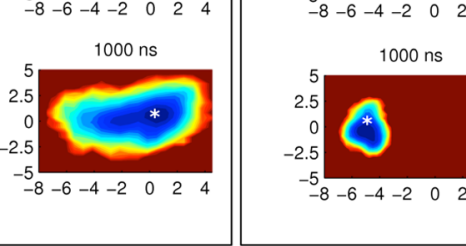

$1000 \mathrm{~ns}$
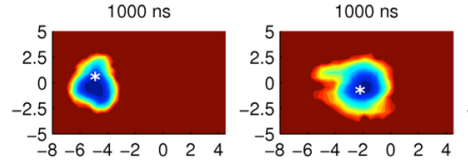

$1000 \mathrm{~ns}$

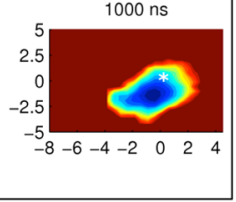

$-k_{B} \operatorname{TIn}\left(N_{i} / N_{o}\right)$

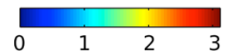

PC $1(\AA)$

Figure 2. Effects of increasing glucose concentration on free MD trajectories of ubiquitin. Logarithmic probability distributions (color-coded as in Figure 1) for projections of the $\mathrm{C} \alpha$ coordinates of ubiquitin at increasing simulation times in water (A), $108 \mathrm{~g} / \mathrm{L}$ glucose (B), and $325 \mathrm{~g} / \mathrm{L}$ glucose (C). $\mathrm{MD}$ simulations in $\mathrm{B}$ and $\mathrm{C}$ were started from structures that correspond to states $\mathrm{L}, \mathrm{C}$, and $\mathrm{R}$ (represented by white asterisks) of the conformational landscape observed in water (Figure 1A). Glucose is abbreviated as "glc".
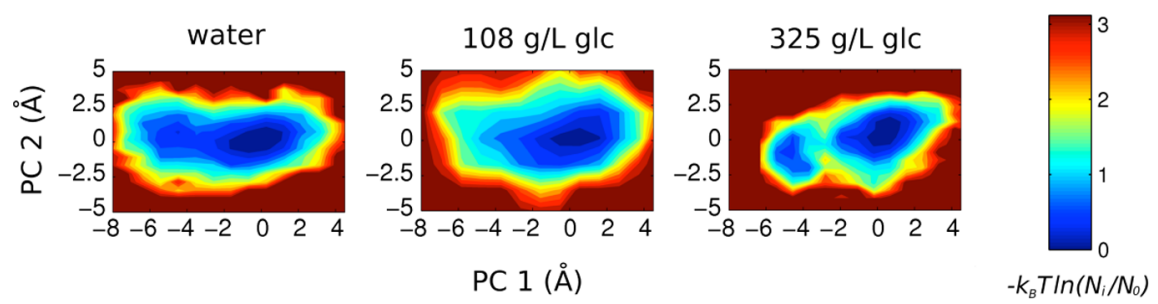

Figure 3. Ubiquitin's conformational space under glucose crowding explored by REMD simulations. Logarithmic probability distributions (colorcoded as in Figure 1A) for the projections of ubiquitin trajectories computed using REMD simulations in water (left), $108 \mathrm{~g} / \mathrm{L}$ glucose (center) and $325 \mathrm{~g} / \mathrm{L}$ glucose (right). Only the series at $301.1 \mathrm{~K}$ was projected. Glucose is abbreviated as "glc".

left and right basins from the conformational landscape, or if they are still present but accessible on longer time scales.

In order to enhance sampling and answer this question, we used REMD simulations as an exploratory tool to probe the conformational space beyond that sampled in the free $\mathrm{MD}$ simulations. The replica-exchange method is one of the few enhanced sampling techniques that do not introduce bias into the simulation and do not require predefined reaction coordinates (more details are given in the Methods section). ${ }^{83}$ We have not used this method to compute full landscapes, but simply to test whether the proteins could gently get out of the basins. From the projections of these simulations (Figure 3, where only the frames at $301.1 \mathrm{~K}$ were projected), it is evident that roughly the whole conformational space observed in water is available in the crowded conditions. [The apparent differences between the three landscapes cannot be regarded as significant, as they are most likely a problem of limited sampling. We provide in the SI text, Figure S11, and Table S6, parameters for the assesment of convergence, which show qualitatively partial convergence only. Yet, this suffices to probe that the protein can escape from its conformational basins under crowding by glucose.] This implies that the lack of a complete conformational landscape in the free MD simulation at $325 \mathrm{~g} / \mathrm{L}$ glucose is only due to a problem of insufficient sampling, and already suggests that crowding increases the energy barriers that separate the basins observed in water, thus slowing down the rate of exploration of the conformational space.

Exploring the Conformational Landscape under Crowded Conditions. The REMD simulations show that conformational states $\mathrm{L}$ and $\mathrm{R}$, although not visited in a simulation started from point $\mathrm{C}$ at $325 \mathrm{~g} / \mathrm{L}$ glucose and only seldom visited at $108 \mathrm{~g} / \mathrm{L}$, do exist in the accessible conformational landscapes under the crowded conditions. However, analysis of the resulting trajectories is not straightforward, complicating the derivation of accurate details about the explored conformational space and its individual basins, especially considering that those under glucose crowding might not have fully converged. In order to improve the description of the landscape easily reachable upon unbiased, unforced $\mathrm{MD}$, we performed standard microsecondlong simulations of ubiquitin in $108 \mathrm{~g} / \mathrm{L}$ and $325 \mathrm{~g} / \mathrm{L}$ glucose, starting from structures that correspond to states $\mathrm{L}$ and $\mathrm{R}$ states of the conformational landscape observed in water (in Figure $1 \mathrm{~A})$.

Starting from state $\mathrm{R}$ at $108 \mathrm{~g} / \mathrm{L}$ glucose (Figure 2B) ubiquitin is able to sample roughly the same conformational 
landscape seen for state C, i.e., that corresponding to the broad rightmost basin of the landscape observed in water. In both simulations, the leftmost basin is explored only once. Starting from state $\mathrm{L}$ in $108 \mathrm{~g} / \mathrm{L}$ glucose, the protein sampled the leftmost basin for $\sim 150 \mathrm{~ns}$, then shifted to the right part of the landscape and spent there most of the remaining simulation time. In the three simulations at $325 \mathrm{~g} / \mathrm{L}$ glucose ubiquitin remains stuck around each starting conformation during the whole simulation time (Figure 3B). This hampers proper computation of the conformational landscapes for these simulations and precludes any accurate estimation of the relative energy of the basins, but for sure indicates that each conformation is very stable against interconversion into other conformations in a submicrosecond time scale, implying very high energetic barriers between them relative to those in water.

Taken together with the replica-exchange data, these results confirm that crowding increases the energy barriers that separate basins (or conformational states) giving place to a much slower conformational exchange. Based on the observation that it takes the simulation in $108 \mathrm{~g} / \mathrm{L}$ glucose 0.5-1 $\mu$ s to explore the same extent of conformational space that is explored in only 50-100 ns of simulation in water, we can very roughly estimate that conformational fluctuations are around an order of magnitude slower already in $108 \mathrm{~g} / \mathrm{L}$ glucose relative to water. The effect at $325 \mathrm{~g} / \mathrm{L}$ is so much stronger that the magnitude of the dampening cannot be estimated at all. Notably, atomic mean square displacements for $\mathrm{C} \alpha$ atoms (Figure S2) show a much softer decrease in the average diffusion of $\mathrm{C} \alpha$ atoms through the conformational space as the concentration of glucose increases, reaching at most a ratio of $\sim 5.5$ when going from 0 to $325 \mathrm{~g} / \mathrm{L}$ (Table S2). This implies that collective motions, i.e., those evident in the trajectory projections, are far more sensitive to glucose-crowded conditions than atomic fluctuations.

Residue-Specific Measurements of Flexibility through $\mathrm{C} \alpha$ Fluctuations and $\mathrm{N}-\mathrm{H}$ Order Parameters. Root-mean square fluctuations (RMSF) and $\mathrm{N}-\mathrm{H}$ order parameters $\left(s^{2}\right)$ measure local motions helping to highlight which protein regions are more affected by a given perturbation, in this case crowded conditions. RMSF profiles computed for the $\mathrm{C} \alpha$ atoms of ubiquitin in all simulations show that loops are the most affected regions (Figures S3A,C). Notably, regions of low RMSF in dilute conditions are unaffected in the crowded media.

$\mathrm{N}-\mathrm{H}$ order parameters are especially interesting because they can be compared directly with the values obtained from NMR relaxation data. Interestingly, calculated order parameters (Figure S3B,D) show reduced mobility of the loops at increasing crowder concentrations, but the effect is milder than that observed in RMSF profiles. This is in agreement with the only experimental measurement of ubiquitin's order parameters in nearly crowded conditions mimicked by micelle encapsulation, which showed mild increases of $s^{2}$ in the loops under confinement. ${ }^{66}$

Both for $\mathrm{C} \alpha$ and $\mathrm{N}-\mathrm{H}$ order parameters, the effects are more important at $325 \mathrm{~g} / \mathrm{L}$ glucose and barely noticeable at $108 \mathrm{~g} / \mathrm{L}$. However, in any case, the predicted effects are of lower magnitude than those reported above for the collective motions that drive exploration of the conformational space, or than the impacts expected on molecular diffusion.

Mechanical Descriptions of the Basins Observed in the Conformational Landscapes. In order to explore the effect of crowding on motions within conformational basins, we computed their mechanical descriptions according to a formalism introduced by Hess, which models the diffusion of a protein through a conformational basin in terms of internal friction-like coefficients $(\eta)$ and harmonic force constants $(k)$ for the principal components of motions inside the basin. For each main basin in each simulation, we analyzed the five first components during $\sim 10-25$ ns-long sections of the trajectories in which the protein remained inside the basin. The full results are given in SI Table S3.

Internal friction coefficients and harmonic force constants turned out to be very sensitive to glucose concentration, for all five principal components of motion. The case of component 1 , taken as an example, shows an exponential increase of its harmonic force constant with glucose concentration leading to sharper and more conformationally restricted basins (Figure 4A). In parallel, internal friction grows roughly linearly with glucose concentration hampering diffusion through the basin (Figure 4B).
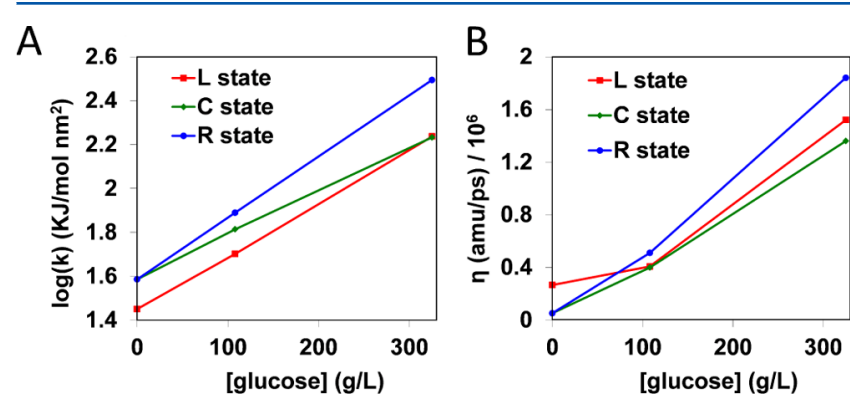

Figure 4. Influence of glucose crowding on the mechanical features of basins in ubiquitin's conformational space. Plots of harmonic force constants $k$ (A) and internal friction coefficients $\eta$ (B) for the first principal component of the main basins explored during free $\mathrm{MD}$ simulations (the remaining components show a similar behavior; see Table S3).

Effect of Crowding on Translational and Rotational Diffusion of Ubiquitin. One of the expected outcomes of crowding is a reduction of the translational and rotational diffusion of solutes-proteins in our case of interest. Works dealing with computational simulations of artificially crowded or cytoplasm-like environments pay close attention to how well experimental translational diffusion is reproduced. ${ }^{93}$ The rotational reorientation time is also related to parameters available from fluorescence and NMR relaxation experiments, thus it should be considered as well. Both rotational and translational diffusion depend inversely on the viscosity of the solution and hence on solute concentrations, but positive or negative deviations are introduced by interacting and/or big solutes, creating special scenarios as described recently. ${ }^{30}$ In vivo measurements and in vitro experiments at physiologically relevant concentrations of crowding agents have shown 5- to 10 -fold reductions in translational and rotational diffusion. $^{30,94-97}$

In the simulations, we observe that the translational diffusion coefficient is reduced by around 50\% in $108 \mathrm{~g} / \mathrm{L}$ glucose and by as much as $80 \%$ (i.e., 5-fold) at $325 \mathrm{~g} / \mathrm{L}$ (Table S4). These numbers imply little deviation from ideality, as observed by NMR experiments for glycerol and proteins as crowding agents. $^{30}$ Given that this ratio is expected to vary among proteins of different sizes, charges and shapes, and on the properties of the crowding agent(s), our results are reasonable. The second-rank rotational relaxation time $\left(\tau_{2}\right)$ computed for 
ubiquitin in the simulation in water is $1.8 \mathrm{~ns}$ (Table S4), which lies on the same time scale of its global correlation time of $(\sim 4$ ns) obtained from NMR data in diluted conditions. ${ }^{98}$ [The second-rank rotational relaxation time (computed from the simulations) and the global correlation time (from model-free analysis of ${ }^{15} \mathrm{~N}$ relaxation data) both relate to the time it takes the protein to make a full rotation. However, it is not clear that they correspond exactly to the same quantity, thus we have avoided any further comparison. The important points are (i) that they are both in the nanoseconds regime, and (ii) that the simulations predict their steep increase (i.e., decrease of rotational diffusion) upon crowding by glucose.] In $108 \mathrm{~g} / \mathrm{L}$ glucose the $\tau_{2}$ increases by 3.7-5.4 times, thus comparing also reasonably against experimental values. At $325 \mathrm{~g} / \mathrm{L}$ glucose, the ratio ranges from 22 to 64 , suggesting a positive deviation from the linear prediction, as observed by NMR for some crowding agents ${ }^{30}$ but somewhat overestimated.

Structure and Dynamics of Water and Glucose Molecules Contacting Ubiquitin. At least two previous works reporting fully atomistic simulations of proteins in crowded media predicted extensive interactions between the small polar molecules and the proteins, which could potentially perturb protein hydration and protein dynamics. ${ }^{16,29}$ In order to characterize the extent of these interactions and explore their impact on ubiquitin's dynamics and hydration, we computed and analyzed the residence times of water and glucose molecules on its surface as a whole and the lifetimes of contacts between water molecules and individual residues of the protein.

The more global analysis, i.e., quantifying interactions between water molecules and the whole protein surface, was based on a formalism that involves the calculation of survival probabilities for protein-solvent/solute interactions followed by analysis of the resulting decay curves. ${ }^{89}$ From a glance at the resulting curves (Figure S4), it is evident that water molecules tend to stay on the protein surface for longer times in the presence of glucose as a crowding agent. This can be quantified by fitting the decay curves to obtain the residence times of water molecules on the surface of the protein on different time scales in the different simulations, as reported in Table S5 and Figure 5. A previous work based on $\sim 10$ ns-long simulations of different proteins in explicit water identified three main exchange regimes for water molecules reaching and leaving the surface of the protein. ${ }^{89}$ Our fit of the survival probabilities in water requires that one more exchange regime be added to account for the full decay of survival probabilities in microsecond-long simulations. In the simulation in water, most water molecules $(\sim 80 \%)$ experience the fastest possible exchange regime with a residence time of 30 ps diffusing slightly off the ideal limit (Kohlrausch's stretching parameter $\gamma$ $=0.899$ against a value of 1 for ideal diffusion, Table S5). Around $17 \%$ of the water molecules are in the second time regime with an average residence time of $140 \mathrm{ps}$, and $2 \%$ have an average residence time of 920 ps corresponding to the third group reported from $10 \mathrm{~ns}$ simulations. Very few molecules $(\sim 0.45 \%)$ have residence times longer than $10 \mathrm{~ns}$, which seems to be the true slowest possible time scale since any time longer than that should be evident in such long simulation.

In the simulations at high glucose concentrations, these molecules interact with the protein removing water molecules from its surface, quickly reducing the average number of water molecules in contact with the protein (Figure 5A). The effect is stronger at higher glucose concentrations and independent of

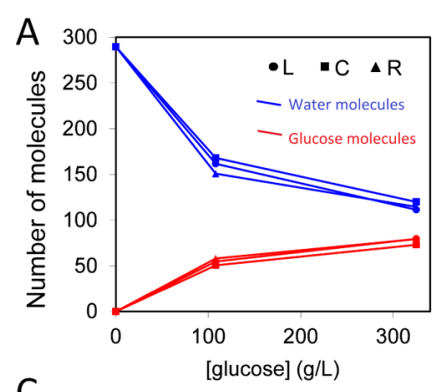

C

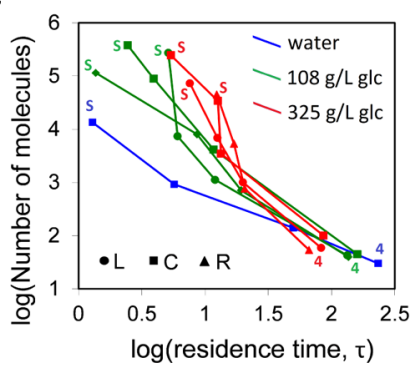

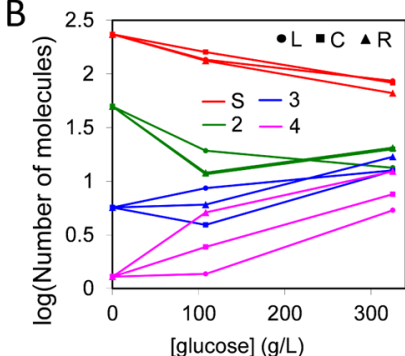

D

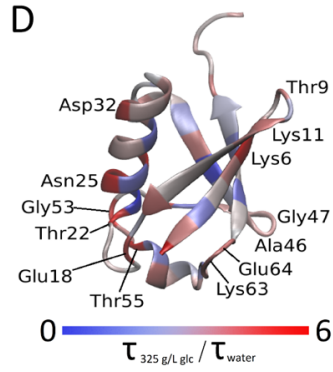

Figure 5. Structure and dynamics of water and glucose on ubiquitin. (A) Average number of water (blue) and glucose (red) molecules interacting with the whole protein throughout the simulations. (B) Number of water molecules exchanging interactions with the protein surface on different time scales: subnanosecond $\left(n_{\mathcal{S}}\right.$, in red), $0.1-100$ ns $\left(n_{2}\right.$ and $n_{3}$, in green and blue), and the slowest process $\left(n_{4}\right.$, pink) reaching up to 380 ns. (C) Number of water molecules under each residence time scale in the seven simulations; each line goes through time scales S, 2, 3, and 4 (for clarity, only $S$ and 4 are shown). Full data corresponding to this analysis is given in Table S5 of the SI. (D) Structure of ubiquitin colored according to the ratio between residence times of water molecules in $325 \mathrm{~g} / \mathrm{L}$ glucose (taken from the simulation started on state $\mathrm{C}$ ) and in water, as computed for individual residues.

the starting conformation. More interestingly, despite the drop in the total number of water molecules wetting the protein surface, the numbers of water molecules with the two longest residence times $\left(n_{3}, n_{4}\right)$ increase with increasing glucose concentration (Figure 5B). Moreover, the residence times of water molecules increase in glucose-crowded conditions (although they are still sampled properly thanks to the lengths of the simulations) (Figure 5C), while the distribution of the number of bound molecules on different time scales shrinks. In turn, the presence of glucose in the solution induces strong non-Brownian diffusion on the fastest time scale of water motions on the protein surface, as revealed by the drop in Kohlrausch parameters. Notice that longer time scales seem to be more affected, so that the shortest one increases at most 3 times (i.e., from $30 \mathrm{ps}$ in water to $100 \mathrm{ps}$ in $325 \mathrm{~g} / \mathrm{L}$ glucose simulated from point $\mathrm{L}$ of the landscape, see Table S5) while the longest increases between 3 and up to 20 times (from $13 \mathrm{~ns}$ in water to $246 \mathrm{~ns}$ in $325 \mathrm{~g} / \mathrm{L}$ simulated from point $\mathrm{L}$ of the landscape).

Analysis of water contacts with individual residues also returns longer average residence times as glucose concentration increases (Figure S5). Protein regions where water residence times are affected the most correspond to flexible segments and very polar/charged exposed residues, followed by structured exposed segments of lower polarity and finally by buried residues, on which water resides for very short times with almost no effect of the crowded conditions (Figure 5D). Put together, all this evidence suggests that binding of glucose molecules to the surface of the protein dehydrates it but traps 
the remaining water molecules, slightly reducing their capacity to jump from residue to residue, and more strongly slowing down their exchange with the bulk. Importantly, these changes in hydration dynamics do not involve changes in the structure of hydration layers, which display conserved features in radial distribution functions (i.e., peaks centered at 2.75 and $3.65 \AA$ as in pure water, perfectly matching with X-rays data ${ }^{99}$ ) and only lowered intensities due to the lower number of molecules wetting the protein (Figure S6A).

Survival probability curves for glucose molecules could not be analyzed under the same formalism employed for water, as most of them led to bad fits. This is possibly due to their very long residence times and hence insufficient sampling of the events as suggested from the survival curves in Figure S4. Notably, there are a few glucose molecules that do not leave the protein surface in the whole simulation times. The lower limit for glucose detaching time scales would hence be around 1-2 orders of magnitude larger than those for water in crowded conditions, explaining their effect in restricting water mobility. Notice from the position of the main peak in the radial distribution function of glucose molecules (Figure S6B) that there is a large density of glucose molecules around the protein, decaying off at longer distances. This means that sugar molecules have been attracted to the protein; indeed, a glance at snapshots from advanced parts of the simulations reveal large, dense, but dynamic cages of sugar molecules formed around the protein, just as predicted by Sidebottom and Tran ${ }^{8}$ and supporting the idea of a water-replacement model for the protective effects of sugars on biomaterials (Figure S7). Notably, the first shell of glucose molecules leaves enough space to accommodate and trap water molecules close to the protein surface. This trapping of water molecules is in line with the observation that the numbers of water molecules in the slowest-exchanging regimes $\left(n_{3}, n_{4}\right)$ increase with glucose concentration, in opposition to the case of fast exchanging waters and the total number of waters.

In one final analysis, we have computed the average number of water and glucose molecules interacting with each residue throughout the simulations (Figure S8). There are no strongly preferential sites for glucose binding and dehydration; in other words, the interactions are unspecific, which is consistent with the chemical structure of sugars and polyols.

Insights from Systems Containing Three Ubiquitin Proteins in a Concentrated Glucose Solution. The large shells of glucose molecules predicted to form around protein molecules could potentially impact properties and processes such as oligomerization or aggregation, i.e., applicable to two or more proteins that make part of the same system. These effects relate in turn to the solubility of the proteins in question and to the effects that intracellular crowders could potentially exert on them. In order to explore these situations, we performed one more set of simulations on systems containing three ubiquitin molecules (one in each of the three states described above) inside cubic boxes of volume $\sim 10^{6} \AA^{3}$ containing either pure water or $325 \mathrm{~g} / \mathrm{L}$ glucose. The estimated protein concentration is around $5 \mathrm{mM}$, at which ubiquitin is expected to dimerize noncovalently by $50 \%$ in a dilute buffer, as shown very recently. ${ }^{100}$

After $250 \mathrm{~ns}$ of simulation at $300 \mathrm{~K}$, the trajectories were analyzed by looking at translational diffusion, internal dynamics, and the structure of water and glucose molecules around the proteins. As observed for the simulations of single proteins, glucose molecules move rapidly forming again cages around the protein molecules (Figure 6A). The resulting dehydration of the proteins' surfaces and the slowdown of internal dynamics
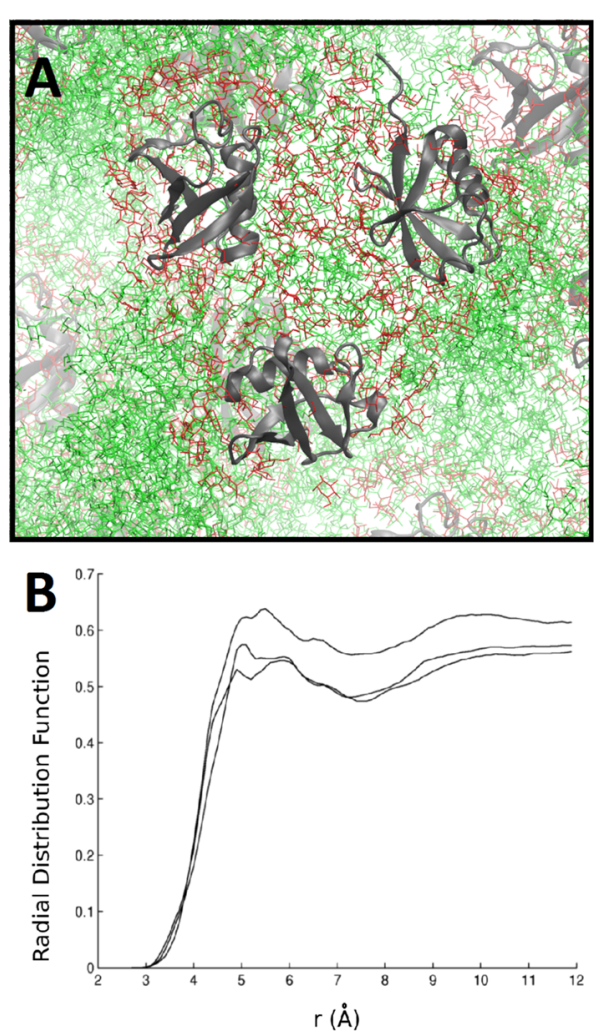

Figure 6. Caging of glucose molecules on ubiquitin. (A) Final snapshot from the simulation of three ubiquitin molecules in glucose proteins are shown as gray cartoons, glucose molecules are shown as sticks (red for those within $8 \AA$ of any protein, green for the rest); water molecules are omitted for clarity. (B) Radial distribution functions of glucose molecules around each protein, averaged throughout the simulation.

are similar to those observed in the simulations involving single protein molecules (Figure S8). The radial distribution functions for glucose molecules show two main shells, also as observed in the simulations of single protein molecules, but with the second shell (peak at around 9-10 ̊) being more dense (Figure 6B). Notably, the second glucose layers of any two proteins overlap partially, and the first layers seem to clash possibly protecting the proteins from aggregation.

The three ubiquitin molecules placed in these systems are far from one another in the starting state, with the minimum distance between any two atoms from different proteins being $\sim 26 \AA$. However, the simulation times are sufficiently long to allow molecules to eventually meet each other as an outcome of diffusion. In fact, two of the protein molecules simulated in water meet at around $170 \mathrm{~ns}$; moreover, they then reorient many times until they form a dimeric structure that remains quite stable for the last $80 \mathrm{~ns}$ of the trajectory. Plots of mean square displacements for the three proteins during the first 150 ns of both simulations (i.e., before the binding event so as to exclude interference from dimerization in the simulation in water) reveal that a ratio of $\sim 5-10$-fold slower diffusion in glucose holds (with an average of $2.1 \times 10^{-11} \mathrm{~m}^{2} / \mathrm{s}$ for the three molecules in water against $3.2 \times 10^{-12}$ for the molecules in glucose) as determined from the simulations of single proteins. 
On the contrary, no protein-protein interactions are observed in the simulation of three protein molecules in a concentrated glucose solution. Although this could be an outcome of limited sampling, which is computationally more demanding on this large system, the description of the glucose structures given above suggests that the first glucose shells prevent direct aggregation of proteins (Figure 6B). This could stand as one contribution of glucose crowding toward enhancing protein stability in solution, i.e., by preventing selfassociation, a prediction that could be tested directly through experiments. Prospective studies should also assess the contributions from slower diffusion itself, alterations in the dielectric constant, and possibly other factors arising from the high concentration of sugar, in suppressing protein aggregation.

One final remark is that the glucose cages that surround the proteins are interconnected, and seem to form dynamic networks that exclude water in the vicinity of the protein, much like in the "noncovalently interacting metabolite structures" proposed by Cossins et al. in their simulation of the bacterial cytosol. ${ }^{29}$ Like in that work and as shown above for single-molecule simulations, these structures are dynamic in that the interacting small molecules are constantly reshuffled so that, on average, all exposed residues contact water and glucose molecules, and there are no protein regions especially prone to interact with one or the other; in other words, the interactions are unspecific.

\section{DISCUSSION}

We have herein looked at the effects of concentrated glucose solutions on the properties of ubiquitin, a small globular protein. One nearly trivial effect of cosolvents and crowders on the kinetic properties of a molecule is a decrease of its translational and rotational diffusion capabilities. This slowdown is reproduced by the simulations, reaching 4- to 5-fold reductions in the translational diffusion coefficients of single proteins at $325 \mathrm{~g} / \mathrm{L}$ glucose relative to diffusion in water, in the range determined experimentally for different crowded systems. $^{30,94-97}$ For rotational diffusion, stronger effects and deviations are predicted by the simulations, which could be due to the simultaneous effect of increasing not only viscosity but also the apparent mass of the protein due to glucose binding and water retardation (see below). These kinds of deviations from linearity are indeed expected for molecules that interact with cosolvents or crowders. ${ }^{30}$ But beyond these effects on diffusion, our simulations show a number of other effects that we discuss in what follows. We observe that sugars cluster irreversibly on ubiquitin molecules within the length of the simulations, consistent with Sidebottom and Tran's prediction that proteins could act as nucleation seeds for the process of sugar clustering. ${ }^{8}$ According to our simulations, this process ends up forming dynamic cages of glucose molecules around the proteins, removing most water molecules from their surfaces but trapping some of them inside a first shell. An important consequence is a generalized slowdown of all collective, large scale internal motions of the proteins, which can in turn modulate processes and properties that depend on internal dynamics.

How Crowding by Small Hydrophilic Molecules Alters the Conformational Landscape of Proteins. In our previous work we reported that proteins simulated under high glucose concentrations were restricted in internal mobility. Our new results allow us to further dissect this effect. First, we observe that the most affected motions are those involving exploration of the conformational space through collective, and thus slower, fluctuations, whereas fast motions are not much dampened. In fact, the reduction observed for the mean squared fluctuations of $\mathrm{C} \alpha$ atoms ( $\mathrm{C} \alpha \mathrm{MSD})$ is similar in magnitude to the drop observed in the translational diffusion coefficient, but the resulting slowdown in the rate of exploration of the conformational space seems to be much stronger (Figures 2 and 3). Indeed, when the concentration of glucose is moderate $(108 \mathrm{~g} / \mathrm{L})$ it takes the protein around $0.5-$ $1 \mu \mathrm{s}$ of simulation time to explore only once the whole conformational space that is explored in only 50-100 ns of simulation in water. This suggests a very roughly estimated $\sim 10$-fold decrease in the rate of exploration of the conformational space at $108 \mathrm{~g} / \mathrm{L}$ glucose, compared to an only 2-fold reduction in the $\mathrm{C} \alpha \mathrm{MSD}$. At $325 \mathrm{~g} / \mathrm{L}$, the slow-down is so much stronger that not even a single transition is observed between two basins within the microsecond time scale, although the replica exchange $\mathrm{MD}$ simulation shows that alternative conformations are still accessible. Finally, projections on increasing principal components show that the effect of crowding decreases too, again indicating that the larger-scale, collective motions are the most affected ones (Figure S9).

One possible explanation for the difference observed in the dampening of local and collective motions is that local fluctuations (to which the C $\alpha$ MSD is sensitive) are dampened to the same extent as overall diffusion is, but dampening the motions of several individual atoms results in a "multiplicative" (i.e., more than simply additive) dampening of their concerted (i.e., collective) motions, which are the ones that lead to effective exploration of the conformational space. This would also explain why the $s^{2}$ and RMSF profiles determined under crowded conditions are not too different from those observed in water, both in simulations and in experimental data, ${ }^{58,65,66}$ with only mild reductions in the flexibility of loops but almost no effect on the structured regions. As a corollary, future experimental investigations on the effect of cosolvents or crowding on internal dynamics should look for differences in microsecond and slower time scales, for example, through deuterium exchange or relaxation dispersion NMR experiments.

The REMD simulations definitely show that conformations similar to those observed in water are accessible in glucosecrowded conditions. Putting these ideas together, we can state that crowding alters the conformational landscape of the protein, but it does not reshape it drastically, keeping roughly the same main conformational states that exist in dilute solution but separating them by higher energy barriers. Going into finer detail, analysis of the individual basins observed in the landscapes further reveals that the conformational space explored by the protein becomes much rougher and deeper in the presence of glucose, and that these two effects increase steeply with glucose concentration. The latter effect results in larger energetic walls around the stable conformations leading to the basins crossing at higher energies. Thus, our results are consistent with a dual effect of crowding on internal dynamics, i.e. (i) imposing higher energy barriers between conformational basins but also (ii) making the basins rougher to structural diffusion. This double effect is depicted schematically in Figure 7, where the "conformational coordinate" is here a generic coordinate that refers not only to exchange between different conformations as in this study of ubiquitin dynamics but also to other coordinates. It is not clear from our studies what the contributions of entropic and enthalpic effects are on each of 


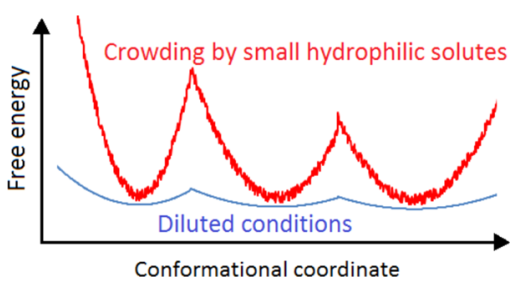

Figure 7. Scheme illustrating our proposition on how crowding by small, polar molecules affects the conformational landscape of proteins, by (i) imposing higher energy barriers between conformational states and (ii) making conformational basins rougher.

these two perturbations of the conformational landscape; these issues deserve further studies.

Among coordinates affected by crowding, those driving protein folding would be important targets of dynamic slowdown by crowding and encapsulation, so as to facilitate protein folding. In this regard, a recent article has briefly commented on how "a sticky cage can slow down folding". ${ }^{101}$

Atomistic Basis for the Conformational Trapping by Crowders. At the atomic level, we attribute the observed effects to the extensive network of interactions established between glucose molecules and the protein surface. We observe that the presence of glucose shells around the protein induces a large dehydration of its surface, but at the same time traps the remaining water molecules. These water molecules jump between residues more slowly than they do in the absence of glucose, but, more importantly, their exchange with bulk water is much hampered under glucose-crowded conditions. This produces an increase in the total number of molecules attached to the protein, dragging its flexible parts. In other words, the long-residing water and glucose molecules become a significant "extension" of the protein, like in the noncovalently interacting metabolite structures proposed to exist in the cytosol. ${ }^{29}$ Similar interpretations of crowding effects as due to increases in the effective mass of the protein have been suggested, highlighting a more important impact on its less compact, i.e., more flexible parts, ${ }^{102}$ just as reported here.

The increase in effective mass can be estimated by considering the trajectory-averaged numbers of water and glucose molecules attached to the protein in each simulation (Table S5): an average of $5.2 \mathrm{kDa}$ of mass coming from water molecules attached to the protein simulated in water; around $12 \mathrm{kDa}$ coming from bound water and glucose molecules are attached in the simulations run at $108 \mathrm{~g} / \mathrm{L}$; and around $23 \mathrm{kDa}$ in the simulations run at $325 \mathrm{~g} / \mathrm{L}$. These numbers are of considerable magnitude relative to ubiquitin's mass of $8.5 \mathrm{kDa}$, and even bigger considering that the loops (whose collective motions determine the conformational landscape) have masses smaller than $1 \mathrm{kDa}$. Finally, the very long residence times of water and glucose molecules on the surface of the protein in the crowded conditions make the effect of increased effective mass even more dramatic.

Potential Impact of Crowding-Perturbed Dynamics on Protein Functional Properties. Although we have dealt here with a simplified model system containing glucose as a crowding agent, the only available atomistic simulation of proteins in media mimicking the bacterial cytosol has suggested the existence of temporal aggregates made up of proteins and metabolites that could induce on proteins effects similar to those reported here. ${ }^{29}$ Based on this analogy, we can extrapolate potential effects of crowding on protein properties important for the cellular biology. Flexibility of the protein backbone and side chains underlies catalytic mechanisms, allosterism, interactions with other proteins, etc. by allowing for the exploration of transient and functionally relevant states. The slow-down induced by crowding on internal motions could kinetically stabilize these transient conformations allowing for example substrates or interacting protein partners to bind to the protein. These effects could thus help to modulate interactions, allosterism, and possibly even catalysis.

Relevance of the Observed Effects Regarding the Roles of Sugars as Preservation Agents and of Small Hydrophilic Molecules on Intracellular Crowding. Interestingly, sugars and polyols in general behave as nearly universal stabilizers of proteins in solution and in dried conditions, protecting them against hot, cold, and even chemical denaturation. This effect is exploited by Nature and also by mankind as a way to preserve biological material, ${ }^{1-3}$ and although it is not completely understood, all explanations link it to the hydration and vitrifying potentials of these small, highly soluble molecules. ${ }^{8-11}$ Our results are consistent with this idea, more specifically with the water-replacement model, which states that glucose molecules replace hydration water removing its entropic effects close to the protein surface at high temperature and favoring vitrification over crystallization processes at cold temperatures. The effects reported here for glucose crowding on hydration patterns further reveal that clustering of glucose molecules around the proteins traps a thin layer of water molecules. Whereas the first shell of glucose molecules could help to prevent aggregation in solution state, the water molecules could keep the protein surface wet inside the glucose cage, which would be of paramount importance to maintain its structure especially in the dried state. Upon cooling, these water molecules would not form a crystal, as their motions are restricted by strong interactions with the immediate layer of glucose molecules. In turn, water molecules located between different protein-glucose clusters could crystallize, but the resulting crystals would be far from the protein molecules; in other words, clustering of sugars in a process nucleated by proteins allows for effective separation of the proteins from the crystalline ice phase.

Our results can also provide a hint to the new view of the bacterial cytosol as a biphasic system made up by a dilute sol and a crowded gel. ${ }^{76}$ Although the intracellular concentrations of sugars are much lower than those employed in the simulations presented here, this is compensated by the presence of many other very hydrophilic molecules which could behave similarly. It is thus possible that processes like those described here, triggered by these kinds of molecules albeit likely to a lower extent, be responsible for favoring their clustering into networks of noncovalently interacting metabolites around proteins. Indeed, atomistic simulations employing types and concentrations of small molecules like those found inside cells have suggested the formation of structures similar to those observed here, ${ }^{29}$ where many small polar molecules cluster around protein molecules and even connect them. These structures could form the gel phase of the cytoplasm, whereas the excluded water would contribute to the sol phase, thus giving place to the biphasic gel-sol state. Inside the gel phase, interactions between proteins and other molecules could be responsible for their stabilization, both thermodynamically and kinetically, as well as for anchoring macromolecules, establishing gradients of different molecules and defining fine-grained compartments inside cells as recently hypothesized. ${ }^{103}$ In this 
regard, $\mathrm{MD}$ simulations have a huge potential for providing quantitative insights into the physics of "sol-gel" phases in the cytoplasm.

\section{CONCLUSION}

We have herein thoroughly described the effects of glucose crowding on the hydration, diffusion, and internal dynamics of ubiquitin as predicted from extensive MD simulations at fully atomistic level. Beyond the basic findings related to protein hydration, internal motions and dynamics, the outcomes of this work have direct implications toward understanding (i) why sugars are such good protein stabilizers, and (ii) the effects of crowding by small hydrophilic molecules on proteins under biological environments. This contribution thus raises a number of new explanations to known phenomena related to the behavior of proteins inside concentrated solutions of polar molecules, as well as new hypotheses about these solutions and about crowded biological media, to be explored by future experiments and simulations.

\section{ASSOCIATED CONTENT}

\section{S Supporting Information}

Supporting Methods and Results: Text describing the methods employed for data analysis; Figure S1 showing different structures of ubiquitin and data mapped on them; Figure S2 with $\mathrm{C} \alpha$ mean square displacements; Figure S3 showing RMSF and $\mathrm{s}^{2}$ profiles; Figure S4 with survival probability plots for water-protein and glucose-protein contacts; Figure S5 with average residence times for water molecules on individual residues; Figure S6 with radial distribution functions for water and glucose molecules; Figure S7 with initial and final snapshots of one simulation in glucose solution; Figure S8 showing the average numbers of water and glucose molecules interacting with each residue; Figure S9 displaying the internal dynamics and hydration structure on the three proteins simulated together; Figure S10 with projections of selected simulations on PCs beyond 2; Figure S11 with plots summarizing data about the extent of sampling in the REMD simulations. Table S1 describing the systems simulated under free dynamics; Table S2 with fits of $\mathrm{C} \alpha$ mean square displacements; Table S3 with parameters describing the conformational basins; Table S4 with translational diffusion and rotational reorientation coefficients; Table S5 with analyses of water-protein and glucose-protein contacts; Table S6 reporting the overlap between potential energy distributions in the REMD simulations. This material is available free of charge via the Internet at http://pubs.acs.org/

\section{AUTHOR INFORMATION}

\section{Corresponding Author}

*E-mail: matteo.dalperaro@epfl.ch.

\section{Author Contributions}

${ }^{\S}$ E.S. and L.A.A. contributed equally.

\section{Notes}

The authors declare no competing financial interest.

\section{ACKNOWLEDGMENTS}

L.A.A. acknowledges EMBO and the Marie Curie Actions for an EMBO Long-Term Postdoctoral Fellowship. Parts of this work were executed on PRACE infrastructures granted to L.A.A. and M.D.P. We thank Paolo De Los Rios, Richard
Lavery, and Bernd Ensing for useful discussions, and the EPFL DIT for technical support.

\section{REFERENCES}

(1) Han, B.; B, J. C. Engineering Challenges in Tissue Preservation. Cell Preserv. Technol. 2004, 2, 91-112.

(2) Storey, K. B.; Storey, J. M. Molecular Biology of Freezing Tolerance. Compr. Physiol. 2013, 3, 1283-1308.

(3) Crowe, J. H.; Carpenter, J. F.; Crowe, L. M. The Role of Vitrification in Anhydrobiosis. Annu. Rev. Physiol. 1998, 60, 73-103.

(4) Penna, T. C. V.; Ishii, M.; Kunimura, J. S.; Cholewa, O. Stability of Recombinant Green Fluorescent Protein (GFPuv) in Glucose Solutions at Different Concentrations and $\mathrm{pH}$ Values. Appl. Biochem. Biotechnol. 2005, 121-124, 501-527.

(5) Imamura, K.; Ogawa, T.; Sakiyama, T.; Nakanishi, K. Effects of Types of Sugar on the Stabilization of Protein in the Dried State. J. Pharm. Sci. 2003, 92, 266-274.

(6) Han, Y.; Jin, B.-S.; Lee, S.-B.; Sohn, Y.; Joung, J.-W.; Lee, J.-H. Effects of Sugar Additives on Protein Stability of Recombinant Human Serum Albumin during Lyophilization and Storage. Arch. Pharm. Res. 2007, 30, 1124-1131.

(7) Back, J. F.; Oakenfull, D.; Smith, M. B. Increased Thermal Stability of Proteins in the Presence of Sugars and Polyols. Biochemistry (Moscow) 1979, 18, 5191-5196.

(8) Sidebottom, D. L.; Tran, T. D. Universal Patterns of Equilibrium Cluster Growth in Aqueous Sugars Observed by Dynamic Light Scattering. Phys. Rev. E: Stat., Nonlinear, Soft Matter Phys. 2010, 82, 051904.

(9) Franks, F. Solid Aqueous Solutions. Pure Appl. Chem. 1993, 65, 2527-2537.

(10) Green, J. L.; Angell, C. A. Phase Relations and Vitrification in Saccharide-Water Solutions and the Trehalose Anomaly. J. Phys. Chem. 1989, 93, 2880-2882.

(11) Crowe, J. H.; Crowe, L. M.; Chapman, D. Preservation of Membranes in Anhydrobiotic Organisms: The Role of Trehalose. Science 1984, 223, 701-703.

(12) McGuffee, S. R; Elcock, A. H. Diffusion, Crowding \& Protein Stability in a Dynamic Molecular Model of the Bacterial Cytoplasm. PLoS Comput. Biol. 2010, 6, e1000694.

(13) Dix, J. A.; Verkman, A. S. Crowding Effects on Diffusion in Solutions and Cells. Annu. Rev. Biophys 2008, 37, 247-263.

(14) Mika, J. T.; Poolman, B. Macromolecule Diffusion and Confinement in Prokaryotic Cells. Curr. Opin Biotechnol 2011, 22, $117-126$.

(15) Wang, Y.; Benton, L. A.; Singh, V.; Pielak, G. J. Disordered Protein Diffusion under Crowded Conditions. J. Phys. Chem. Lett. 2012, 3, 2703-2706.

(16) Abriata, L. A.; Spiga, E.; Dal Peraro, M. All-Atom Simulations of Crowding Effects on Ubiquitin Dynamics. Phys. Biol. 2013, 10, 045006 .

(17) Caffarena, E. R.; Grigera, J. R. Hydration of Glucose in the Rubbery and Glassy States Studied by Molecular Dynamics Simulation. Carbohydr. Res. 1999, 315, 63-69.

(18) Caffarena, E. R.; Grigera, J. R. Glass Transition in Aqueous Solutions of Glucose. Molecular Dynamics Simulation. Carbohydr. Res. 1997, 300, 51-57.

(19) Roberts, C. J.; Debenedetti, P. G. Structure and Dynamics in Concentrated, Amorphous Carbohydrate-Water Systems by Molecular Dynamics Simulation. J. Phys. Chem. B 1999, 103, 7308-7318.

(20) Ekdawi-Sever, N.; de Pablo, J. J.; Feick, E.; von Meerwall, E. Diffusion of Sucrose and $\alpha, \alpha$-Trehalose in Aqueous Solutions. J. Phys. Chem. A 2003, 107, 936-943.

(21) Lerbret, A.; Bordat, P.; Affouard, F.; Descamps, M.; Migliardo, F. How Homogeneous Are the Trehalose, Maltose, and Sucrose Water Solutions? An Insight from Molecular Dynamics Simulations. J. Phys. Chem. B 2005, 109, 11046-11057.

(22) Sidebottom, D. L. Ultraslow Relaxation of Hydrogen-Bonded Dynamic Clusters in Glass-Forming Aqueous Glucose Solutions: A 
Light Scattering Study. Phys. Rev. E: Stat., Nonlinear, Soft Matter Phys. 2007, 76, 011505.

(23) Pomata, M. H. H.; Sonoda, M. T.; Skaf, M. S.; Elola, M. D. Anomalous Dynamics of Hydration Water in Carbohydrate Solutions. J. Phys. Chem. B 2009, 113, 12999-13006.

(24) Bennett, B. D.; Kimball, E. H.; Gao, M.; Osterhout, R.; Van Dien, S. J.; Rabinowitz, J. D. Absolute Metabolite Concentrations and Implied Enzyme Active Site Occupancy in Escherichia coli. Nat. Chem. Biol. 2009, 5, 593-599.

(25) Zhou, H. X.; Rivas, G.; Minton, A. P. Macromolecular Crowding and Confinement: Biochemical, Biophysical, and Potential Physiological Consequences. Annu. Rev. Biophys. 2008, 37, 375-397.

(26) Gershenson, A.; Gierasch, L. M. Protein Folding in the Cell: Challenges and Progress. Curr. Opin. Struct. Biol. 2011, 21, 32-41.

(27) Zhou, H. X. Effect of Mixed Macromolecular Crowding Agents on Protein Folding. Proteins 2008, 72, 1109-1113.

(28) Yuan, J. M.; Chyan, C. L.; Zhou, H. X.; Chung, T. Y.; Peng, H.; Ping, G.; Yang, G. The Effects of Macromolecular Crowding on the Mechanical Stability of Protein Molecules. Protein Sci. 2008, 17, 21562166.

(29) Cossins, B. P.; Jacobson, M. P.; Guallar, V. A New View of the Bacterial Cytosol Environment. PLoS Comput. Biol. 2011, 7, e1002066.

(30) Wang, Y.; Li, C.; Pielak, G. J. Effects of Proteins on Protein Diffusion. J. Am. Chem. Soc. 2010, 132, 9392-9397.

(31) Waudby, C. A.; Mantle, M. D.; Cabrita, L. D.; Gladden, L. F.; Dobson, C. M.; Christodoulou, J. Rapid Distinction of Intracellular and Extracellular Proteins Using NMR Diffusion Measurements. J. Am. Chem. Soc. 2012, 134, 11312-11315.

(32) Martorell, G.; Adrover, M.; Kelly, G.; Temussi, P. A.; Pastore, A. A Natural and Readily Available Crowding Agent: NMR Studies of Proteins in Hen Egg White. Proteins 2011, 79, 1408-1415.

(33) Miklos, A. C.; Sarkar, M.; Wang, Y.; Pielak, G. J. Protein Crowding Tunes Protein Stability. J. Am. Chem. Soc. 2011, 133, 71167120.

(34) Pozdnyakova, I.; Wittung-Stafshede, P. Non-Linear Effects of Macromolecular Crowding on Enzymatic Activity of Multi-Copper Oxidase. Biochim. Biophys. Acta 2010, 1804, 740-744.

(35) Wang, Y.; Sarkar, M.; Smith, A. E.; Krois, A. S.; Pielak, G. J. Macromolecular Crowding and Protein Stability. J. Am. Chem. Soc. 2012, 134, 16614-16618.

(36) Benton, L. A.; Smith, A. E.; Young, G. B.; Pielak, G. J. Unexpected Effects of Macromolecular Crowding on Protein Stability. Biochemistry 2012, 51, 9773-9775.

(37) Van Horn, W. D.; Ogilvie, M. E.; Flynn, P. F. Reverse Micelle Encapsulation as a Model for Intracellular Crowding. J. Am. Chem. Soc. 2009, 131, 8030-8039.

(38) Wang, Q.; Liang, K. C.; Czader, A.; Waxham, M. N.; Cheung, M. S. The Effect of Macromolecular Crowding, Ionic Strength and Calcium Binding on Calmodulin Dynamics. PLoS Comput. Biol. 2011, 7, e1002114.

(39) Feig, M.; Sugita, Y. Variable Interactions between Protein Crowders and Biomolecular Solutes Are Important in Understanding Cellular Crowding. J. Phys. Chem. B 2012, 116, 599-605.

(40) Harada, R.; Sugita, Y.; Feig, M. Protein Crowding Affects Hydration Structure and Dynamics. J. Am. Chem. Soc. 2012, 134, 4842-4849.

(41) Homouz, D.; Stagg, L.; Wittung-Stafshede, P.; Cheung, M. S. Macromolecular Crowding Modulates Folding Mechanism of Alpha/ Beta Protein Apoflavodoxin. Biophys. J. 2009, 96, 671-680.

(42) Dhar, A.; Samiotakis, A.; Ebbinghaus, S.; Nienhaus, L.; Homouz, D.; Gruebele, M.; Cheung, M. S. Structure, Function, and Folding of Phosphoglycerate Kinase Are Strongly Perturbed by Macromolecular Crowding. Proc. Natl. Acad. Sci. U. S. A. 2010, 107, 17586-17591.

(43) Zimmerman, S. B.; Trach, S. O. Estimation of Macromolecule Concentrations and Excluded Volume Effects for the Cytoplasm of Escherichia coli. J. Mol. Biol. 1991, 222, 599-620.

(44) Minton, A. P. The Influence of Macromolecular Crowding and Macromolecular Confinement on Biochemical Reactions in Physiological Media. J. Biol. Chem. 2001, 276, 10577-10580.
(45) Tian, J.; Garcia, A. E. Simulations of the Confinement of Ubiquitin in Self-Assembled Reverse Micelles. J. Chem. Phys. 2011, 134, 225101.

(46) Bicout, D. J.; Field, M. J. Stochastic Dynamics Simulations of Macromolecular Diffusion in a Model of the Cytoplasm of Escherichia coli. J. Phys. Chem. 1996, 100, 2489-2497.

(47) Ridgway, D.; Broderick, G.; Lopez-Campistrous, A.; Ru’aini, M.; Winter, P.; Hamilton, M.; Boulanger, P.; Kovalenko, A.; Ellison, M. J. Coarse-Grained Molecular Simulation of Diffusion and Reaction Kinetics in a Crowded Virtual Cytoplasm. Biophys. J. 2008, 94, 37483759.

(48) Ando, T.; Skolnick, J. Crowding and Hydrodynamic Interactions Likely Dominate in Vivo Macromolecular Motion. Proc. Natl. Acad. Sci. U. S. A. 2010, 107, 18457-18462.

(49) Pielak, G. J.; Li, C.; Miklos, A. C.; Schlesinger, A. P.; Slade, K. M.; Wang, G. F.; Zigoneanu, I. G. Protein Nuclear Magnetic Resonance under Physiological Conditions. Biochemistry 2009, 48, 226-234.

(50) Selenko, P.; Wagner, G. Looking into Live Cells with in-Cell NMR Spectroscopy. J. Struct. Biol. 2007, 158, 244-253.

(51) Serber, Z.; Selenko, P.; Hansel, R.; Reckel, S.; Lohr, F.; Ferrell, J. E.; Wagner, G.; Dotsch, V. Investigating Macromolecules inside Cultured and Injected Cells by in-Cell NMR Spectroscopy. Nat. Protoc. 2006, 1, 2701-2709.

(52) Burz, D. S.; Dutta, K.; Cowburn, D.; Shekhtman, A. In-Cell NMR for Protein-Protein Interactions (STINT-NMR). Nat. Protoc. 2006, 1, 146-152.

(53) Burz, D. S.; Dutta, K.; Cowburn, D.; Shekhtman, A. Mapping Structural Interactions Using in-Cell NMR Spectroscopy (STINTNMR). Nat. Methods 2006, 3, 91-93.

(54) Inomata, K.; Ohno, A.; Tochio, H.; Isogai, S.; Tenno, T.; Nakase, I.; Takeuchi, T.; Futaki, S.; Ito, Y.; Hiroaki, H.; et al. HighResolution Multi-Dimensional NMR Spectroscopy of Proteins in Human Cells. Nature 2009, 458, 106-109.

(55) Sakakibara, D.; Sasaki, A.; Ikeya, T.; Hamatsu, J.; Hanashima, T.; Mishima, M.; Yoshimasu, M.; Hayashi, N.; Mikawa, T.; Walchli, M.; et al. Protein Structure Determination in Living Cells by in-Cell NMR Spectroscopy. Nature 2009, 458, 102-105.

(56) Binolfi, A.; Theillet, F. X.; Selenko, P. Bacterial in-Cell NMR of Human Alpha-Synuclein: A Disordered Monomer by Nature? Biochem. Soc. Trans. 2012, 40, 950-954.

(57) Foffi, G.; Pastore, A.; Piazza, F.; Temussi, P. A. Macromolecular Crowding: Chemistry and Physics Meet Biology (Ascona, Switzerland, 10-14 June 2012). Phys. Biol. 2013, 10, 1-5.

(58) Latham, M. P.; Kay, L. E. Is Buffer a Good Proxy for a Crowded Cell-Like Environment? A Comparative NMR Study of Calmodulin Side-Chain Dynamics in Buffer and E. coli Lysate. PLoS One 2012, 7, e48226.

(59) Miklos, A. C.; Li, C.; Sharaf, N. G.; Pielak, G. J. Volume Exclusion and Soft Interaction Effects on Protein Stability under Crowded Conditions. Biochemistry (Moscow) 2010, 49, 6984-6991.

(60) Dedmon, M. M.; Patel, C. N.; Young, G. B.; Pielak, G. J. FlgM Gains Structure in Living Cells. Proc. Natl. Acad. Sci. U. S. A. 2002, 99, 12681-12684.

(61) Sasahara, K.; McPhie, P.; Minton, A. P. Effect of Dextran on Protein Stability and Conformation Attributed to Macromolecular Crowding. J. Mol. Biol. 2003, 326, 1227-1237.

(62) Christiansen, A.; Wang, Q.; Samiotakis, A.; Cheung, M. S.; Wittung-Stafshede, P. Factors Defining Effects of Macromolecular Crowding on Protein Stability: An in Vitro/in Silico Case Study Using Cytochrome c. Biochemistry (Moscow) 2010, 49, 6519-6530.

(63) Stagg, L.; Zhang, S. Q.; Cheung, M. S.; Wittung-Stafshede, P. Molecular Crowding Enhances Native Structure and Stability of Alpha/Beta Protein Flavodoxin. Proc. Natl. Acad. Sci. U. S. A. 2007, 104, 18976-18981.

(64) Perham, M.; Stagg, L.; Wittung-Stafshede, P. Macromolecular Crowding Increases Structural Content of Folded Proteins. FEBS Lett. 2007, 581, 5065-5069. 
(65) Cino, E. A.; Karttunen, M.; Choy, W.-Y. Effects of Molecular Crowding on the Dynamics of Intrinsically Disordered Proteins. PLoS One 2012, 7, e49876.

(66) Simorellis, A. K.; Flynn, P. F. Fast Local Backbone Dynamics of Encapsulated Ubiquitin. J. Am. Chem. Soc. 2006, 128, 9580-9581.

(67) Tjandra, N.; Feller, S. E.; Pastor, R. W.; Bax, A. Rotational Diffusion Anisotropy of Human Ubiquitin from ${ }^{15} \mathrm{~N}$ NMR Relaxation. J. Am. Chem. Soc. 1995, 117, 12562-12565.

(68) Lakomek, N. A.; Carlomagno, T.; Becker, S.; Griesinger, C.; Meiler, J. A Thorough Dynamic Interpretation of Residual Dipolar Couplings in Ubiquitin. J. Biomol NMR 2006, 34, 101-115.

(69) Lakomek, N. A.; Walter, K. F.; Fares, C.; Lange, O. F.; de Groot, B. L.; Grubmuller, H.; Bruschweiler, R.; Munk, A.; Becker, S.; Meiler, J.; et al. Self-Consistent Residual Dipolar Coupling Based Model-Free Analysis for the Robust Determination of Nanosecond to Microsecond Protein Dynamics. J. Biomol NMR 2008, 41, 139-155.

(70) Lange, O. F.; van der Spoel, D.; de Groot, B. L. Scrutinizing Molecular Mechanics Force Fields on the Submicrosecond Timescale with NMR Data. Biophys. J. 2010, 99, 647-655.

(71) Lakomek, N. A.; Lange, O. F.; Walter, K. F.; Fares, C.; Egger, D.; Lunkenheimer, P.; Meiler, J.; Grubmuller, H.; Becker, S.; de Groot, B. L.; et al. Residual Dipolar Couplings as a Tool to Study Molecular Recognition of Ubiquitin. Biochem. Soc. Trans. 2008, 36, 1433-1437.

(72) Lange, O. F.; Lakomek, N. A.; Fares, C.; Schroder, G. F.; Walter, K. F.; Becker, S.; Meiler, J.; Grubmuller, H.; Griesinger, C.; de Groot, B. L. Recognition Dynamics up to Microseconds Revealed from an RDC-Derived Ubiquitin Ensemble in Solution. Science 2008, 320, $1471-1475$

(73) Ruan, K.; Tolman, J. R. Composite Alignment Media for the Measurement of Independent Sets of NMR Residual Dipolar Couplings. J. Am. Chem. Soc. 2005, 127, 15032-15033.

(74) Maragakis, P.; Lindorff-Larsen, K.; Eastwood, M. P.; Dror, R. O.; Klepeis, J. L.; Arkin, I. T.; Jensen, M. O.; Xu, H.; Trbovic, N.; Friesner, R. A.; et al. Microsecond Molecular Dynamics Simulation Shows Effect of Slow Loop Dynamics on Backbone Amide Order Parameters of Proteins. J. Phys. Chem. B 2008, 112, 6155-6158.

(75) Chen, C.; Li, W. Z.; Song, Y. C.; Weng, L. D.; Zhang, N. Formation of Water and Glucose Clusters by Hydrogen Bonds in Glucose Aqueous Solutions. Comput. Theor. Chem. 2012, 984, 85-92.

(76) Spitzer, J.; Poolman, B. How Crowded Is the Prokaryotic Cytoplasm? FEBS Lett. 2013, 587, 2094-2098.

(77) Phillips, J. C.; Braun, R; Wang, W.; Gumbart, J.; Tajkhorshid, E.; Villa, E.; Chipot, C.; Skeel, R. D.; Kalé, L.; Schulten, K. Scalable Molecular Dynamics with NAMD. J. Comput. Chem. 2005, 26, 17811802.

(78) Hornak, V.; Abel, R.; Okur, A.; Strockbine, B.; Roitberg, A.; Simmerling, C. Comparison of Multiple Amber Force Fields and Development of Improved Protein Backbone Parameters. Proteins 2006, 65, 712-725.

(79) Jorgensen, W. L.; Chandrasekhar, J.; Madura, J. D.; Impey, R. W.; Klein, M. L. Comparison of Simple Potential Functions for Simulating Liquid Water. J. Chem. Phys. 1983, 79, 926-935.

(80) Kirschner, K. N.; Yongye, A. B.; Tschampel, S. M.; GonzalezOuteirino, J.; Daniels, C. R.; Foley, B. L.; Woods, R. J. GLYCAM06: A Generalizable Biomolecular Force Field. Carbohydrates. J. Comput. Chem. 2008, 29, 622-655.

(81) Cornilescu, G.; Marquardt, J. L.; Ottiger, M.; Bax, A. Validation of Protein Structure from Anisotropic Carbonyl Chemical Shifts in a Dilute Liquid Crystalline Phase. J. Am. Chem. Soc. 1998, 120, 68366837.

(82) Martinez, L.; Andrade, R.; Birgin, E. G.; Martinez, J. M. Packmol: A Package for Building Initial Configurations for Molecular Dynamics Simulations. J. Comput. Chem. 2009, 30, 2157-2164.

(83) Sugita, Y.; Okamoto, Y. Replica-Exchange Molecular Dynamics Method for Protein Folding. Chem. Phys. Lett. 1999, 314, 141-151.

(84) Case, D. A.; Cheatham, T. E.; Darden, T.; Gohlke, H.; Luo, R.; Merz, K. M.; Onufriev, A.; Simmerling, C.; Wang, B.; Woods, R. J. The Amber Biomolecular Simulation Programs. J. Comput. Chem. 2005, 26, $1668-1688$.
(85) Rosta, E.; Buchete, N. V.; Hummer, G. Thermostat Artifacts in Replica Exchange Molecular Dynamics Simulations. J. Chem. Theory Comput. 2009, 5, 1393-1399.

(86) Sindhikara, D.; Meng, Y.; Roitberg, A. E. Exchange Frequency in Replica Exchange Molecular Dynamics. J. Chem. Phys. 2008, 128, 024103.

(87) Hess, B. Similarities between Principal Components of Protein Dynamics and Random Diffusion. Phys. Rev. E 2000, 62, 8438-8448.

(88) Marchi, M.; Sterpone, F.; Ceccarelli, M. Water Rotational Relaxation and Diffusion in Hydrated Lysozyme. J. Am. Chem. Soc. 2002, 124, 6787-6791.

(89) Sterpone, F.; Ceccarelli, M.; Marchi, M. Dynamics of Hydration in Hen Egg White Lysozyme. J. Mol. Biol. 2001, 311, 409-419.

(90) Long, D.; Bruschweiler, R. In Silico Elucidation of the Recognition Dynamics of Ubiquitin. PLoS Comput. Biol. 2011, 7, e1002035.

(91) Peters, J. H.; de Groot, B. L. Ubiquitin Dynamics in Complexes Reveal Molecular Recognition Mechanisms beyond Induced Fit and Conformational Selection. PLoS Comput. Biol. 2012, 8, e1002704.

(92) Wlodarski, T.; Zagrovic, B. Conformational Selection and Induced Fit Mechanism Underlie Specificity in Noncovalent Interactions with Ubiquitin. Proc. Natl. Acad. Sci. U. S. A. 2009, 106, 19346-19351.

(93) Frembgen-Kesner, T.; Elcock, A. H. Computer Simulations of the Bacterial Cytoplasm. Biophys. Rev. 2013, 5, 109-119.

(94) Elowitz, M. B.; Surette, M. G.; Wolf, P. E.; Stock, J. B.; Leibler, S. Protein Mobility in the Cytoplasm of Escherichia coli. J. Bacteriol. 1999, 181, 197-203.

(95) Konopka, M. C.; Sochacki, K. A.; Bratton, B. P.; Shkel, I. A.; Record, M. T.; Weisshaar, J. C. Cytoplasmic Protein Mobility in Osmotically Stressed Escherichia coli. J. Bacteriol. 2009, 191, 231-237.

(96) Konopka, M. C.; Shkel, I. A.; Cayley, S.; Record, M. T.; Weisshaar, J. C. Crowding and Confinement Effects on Protein Diffusion in Vivo. J. Bacteriol. 2006, 188, 6115-6123.

(97) Mullineaux, C. W.; Nenninger, A.; Ray, N.; Robinson, C. Diffusion of Green Fluorescent Protein in Three Cell Environments in Escherichia coli. J. Bacteriol. 2006, 188, 3442-3448.

(98) Lee, A. L.; Wand, A. J. Assessing Potential Bias in the Determination of Rotational Correlation Times of Proteins by NMR Relaxation. J. Biomol NMR 1999, 13, 101-112.

(99) Chen, X.; Weber, I.; Harrison, R. W. Hydration Water and Bulk Water in Proteins Have Distinct Properties in Radial Distributions Calculated from 105 Atomic Resolution Crystal Structures. J. Phys. Chem. B 2008, 112, 12073-12080.

(100) Liu, Z.; Zhang, W.-P.; Xing, Q.; Ren, X.; Liu, M.; Tang, C. Noncovalent Dimerization of Ubiquitin. Angew. Chem., Int. Ed. Engl. 2012, 51, 469-472.

(101) Boomsma, W.; Lindorff-Larsen, K. A Sticky Cage Can Slow down Folding. Biophys. J. 2013, 104, 964-965.

(102) Miklos, A. C.; Conggang, L.; Pielak, G. J. Using NMRDetected Backbone Amide 1H Exchange to Assess Macromolecular Crowding Effects on Globular-Protein Stability. Methods Enzym. 2009, $466,1-17$.

(103) Spitzer, J.; Poolman, B. The Role of Biomacromolecular Crowding, Ionic Strength, and Physicochemical Gradients in the Complexities of Life's Emergence. Microbiol. Mol. Biol. Rev. 2009, 73, 371-388. 